\title{
Mathematical modeling of horizontal drillstrings subjected to friction and shocks effects
}

\author{
$\underline{\text { Americo Cunha Jr}}^{1,2} \quad$ Christian Soize ${ }^{2} \quad$ Rubens Sampaio $^{1}$ \\ ${ }^{1}$ PUC-Rio, Department of Mechanical Engineering, Brazil \\ ${ }^{2}$ Université Paris-Est, Laboratory of Multi-Scale Modeling and Simulation, France \\ e-mail: americo.cunhajr@gmail.com christian.soize@univ-paris-est.fr rsampaio@puc-rio.br
}

\begin{abstract}
During the process of oil prospection, an equipment called drillstring is used to drill the soil. This device presents a three-dimensional nonlinear dynamics subjected to effects of friction and shock. This work aims to analyze the nonlinear dynamics of a drillstring in horizontal configuration. For this purpose, the drillstring is modeled as a beam theory with rotatory inertia and shear deformation of the the cross section, undergoing large displacements. This model also takes into account the effects of friction and shock, induced by the lateral impacts between the drillstring and the borehole wall. The model equations are discretized using the Galerkin/finite element method, and the resulting initial value problem is integrated using the Newmark method. Numerical simulations are conducted to investigate the effects of the lateral shock in nonlinear dynamic of the column.
\end{abstract}

Key-words: nonlinear dynamics, finite element method, numerical analysis

\section{Introduction}

Oil prospecting uses an equipment called drillstring to drill the soil until the reservoir level. This equipment is a long column under rotation, composed by a sequence of connected drillpipes and auxiliary equipment. The dynamics of this column is very complex because, under normal operational conditions, it is subjected to longitudinal, lateral and torsional vibrations, which present a nonlinear coupling, and the structure is also subjected to shocks due to the drillpipes/borehole impacts [12]. The dynamics of the column in a vertical configuration, has been the subject of several scientific papers, [11, 13, 8, 17, but to the best of the authors' knowledge, only one work in the open literature studies the dynamics of this column in horizontal configuration [6].

This work intends to analyze the three-dimensional nonlinear dynamics of a drillstring in horizontal configuration. For this purpose, it is presented a deterministic computational model which uses a nonlinear beam theory that takes into account the rotatory inertia and shear deformation of the beam cross section, as well as the coupling between longitudinal, transverse and torsional vibrations. This model also includes the effects of torsional friction and lateral shocks induced by the impacts between the drillstring and the borehole wall. Numerical simulations are conducted in order to investigate the influence of the lateral shocks in the nonlinear dynamic behavior the mechanical system under analysis.

The organization of the paper is as follows. The section 2 presents the mathematical modeling of the physical system of interest. In the section 3 are presented the numerical results of simulations which were performed to analyze the nonlinear dynamics of the horizontal drillstring. Finally, in the section 4, the conclusions of the work are reemphasized, and some paths for future works are pointed out. 


\section{Mathematical modeling}

\subsection{Mechanical system of interest}

The bottom part of the horizontal drillstring is modeled as an annular beam, blocked to move transversely in both extremes, and blocked to rotate transversely in the left extreme. This beam is free to rotate around the $x$ axis, and to move longitudinally. It has a length $L$, cross section area $A$, and is made of a material with mass density $\rho$, elastic modulus $E$, and shear modulus $G$. Due to the horizontal configuration, it is subject to a gravitational field, which induces an acceleration $g$. It loses energy through a mechanism of viscous dissipation, proportional to the mass operator, with damping coefficient $c$. A sketch of this physical system can be seen in Figure 1.

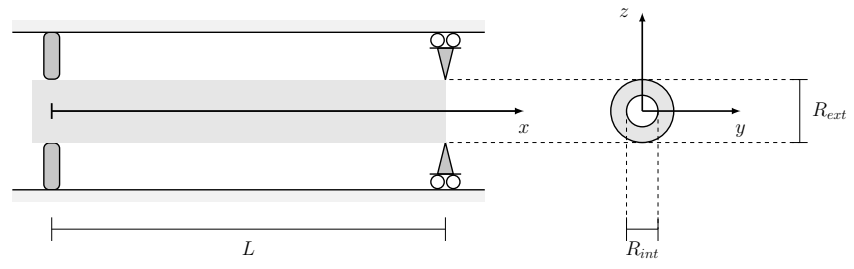

Figure 1: Sketch of the beam used to model the bottom part of the horizontal drillstring.

In the beam model used in this work, it is supposed small rotations in the transversal directions and large displacements for the beam neutral fiber displacements. The displacements of a beam neutral fiber point in $x, y$, and $z$ directions are, respectively, denoted by $u, v$, and $w$, while $\theta_{x}, \theta_{y}$, and $\theta_{z}$ represent rotations of the beam around the $x, y$, and $z$ axes respectively. These physical quantities depend on the position $x$ and the time $t$.

\section{$2.2 \quad$ Friction and shock effects}

The effects of normal shock and torsional friction between the beam and the borehole wall are modeled in terms of a measure of penetration in the wall of a beam cross section [3], dubbed indentation, which is defined as

$$
\delta_{F S}=r-\text { gap, }
$$

where the lateral displacement of the neutral fiber is

$$
r=\sqrt{v^{2}+w^{2}},
$$

and gap denotes the spacing between the undeformed beam and the borehole wall. One has that $\delta_{F S}>0$ in case of an impact, or $\delta_{F S} \leq 0$ otherwise, as can be seen in Figure 2 .
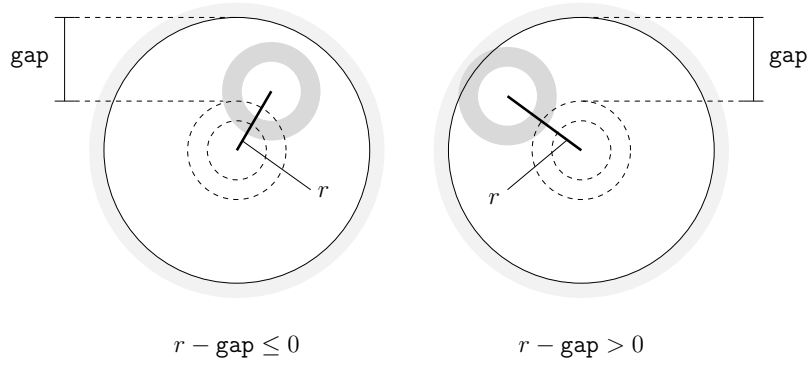

Figure 2: Indentation parameter in a situation without impact (left) or with impact (right). 
When an impact occurs, act on the beam cross section a normal force of the form

$$
F_{F S}=-k_{F S_{1}} \delta_{F S}-k_{F S_{2}} \delta_{F S}^{3}-c_{F S}|\delta|^{3} \dot{\delta}_{F S}
$$

and a Coulomb frictional torque of the form

$$
T_{F S}=-\mu_{F S} F_{F S} R_{b h} \operatorname{sgn}\left(\dot{\theta}_{x}\right) .
$$

In the above equations, $k_{F S_{1}}, k_{F S_{2}}$ and $c_{F S}$ are constants of the Hunt and Crossley shock model [5], while $\mu_{F S}$ is a friction coefficient, $R_{b h}$ is the borehole radius, and $\operatorname{sgn}(\cdot)$ the sign function. For simplicity, it is adopted the hypothesis that the mechanical contact between the beam and the borehole wall occurs exactly in the nodes of the finite element mesh.

\subsection{Weak formulation of the nonlinear dynamics}

Starting from a modified version of the extended Hamilton's principle, to include the effects of dissipation, one can write the weak form ${ }^{1}$ of the nonlinear equation of motion of the system as

$$
\mathcal{M}(\psi, \ddot{\boldsymbol{U}})+\mathcal{C}(\boldsymbol{\psi}, \dot{\boldsymbol{U}})+\mathcal{K}(\boldsymbol{\psi}, \boldsymbol{U})=\mathcal{F}_{N L}(\boldsymbol{\psi}, \boldsymbol{U}, \dot{\boldsymbol{U}}, \ddot{\boldsymbol{U}})
$$

valid for any "suitable" $\boldsymbol{\psi}$, and being $\mathcal{M}$ the mass operator, $\mathcal{C}$ the damping operator, $\mathcal{K}$ the stiffness operator, and $\mathcal{F}_{N L}$ the nonlinear force operator. Also, the field variables and their weight functions are lumped in the vectors fields

$$
\boldsymbol{U}=\left(\begin{array}{c}
u \\
v \\
w \\
\theta_{x} \\
\theta_{y} \\
\theta_{z}
\end{array}\right), \quad \text { and } \quad \boldsymbol{\psi}=\left(\begin{array}{c}
\psi_{u} \\
\psi_{v} \\
\psi_{w} \\
\psi_{\theta_{x}} \\
\psi_{\theta_{y}} \\
\psi_{\theta_{z}}
\end{array}\right)
$$

The weak form of the initial conditions reads

$$
\mathcal{M}(\boldsymbol{\psi}, \boldsymbol{U}(0))=\mathcal{M}\left(\boldsymbol{\psi}, \boldsymbol{U}_{0}\right)
$$

and

$$
\mathcal{M}(\boldsymbol{\psi}, \dot{\boldsymbol{U}}(0))=\mathcal{M}\left(\boldsymbol{\psi}, \dot{\boldsymbol{U}}_{0}\right)
$$

where $\boldsymbol{U}_{0}$ and $\dot{\boldsymbol{U}}_{0}$, respectively, denote the initial displacement, and the initial velocity fields.

The operators that appear in Eqs.(5), (7), and (8) are defined by complex expressions, which are not presented in this article for reasons of space limitation. The interested reader can find then in [1] and [2].

\subsection{Discretization of the model equations}

The Galerkin/finite element method [4] is employed to construct an approximation to the solution of the boundary/initial value problem corresponding to the weak formulation given by Eqs.(5), (7) and (8).

\footnotetext{
${ }^{1}$ It is implicitly assumed that, all the hypothesis necessary to, the initial-boundary value problem defined by the Eqs. (5), (7), and (8), be well posed are satisfied.
} 
In this way, one gets an initial value problem of the form

$$
\begin{gathered}
{[\mathcal{M}] \ddot{\boldsymbol{Q}}(t)+[\mathcal{C}] \dot{\boldsymbol{Q}}(t)+[\mathcal{K}] \boldsymbol{Q}(t)=\mathcal{F}_{N L}(\boldsymbol{Q}(t), \dot{\boldsymbol{Q}}(t), \ddot{\boldsymbol{Q}}(t)),} \\
\boldsymbol{Q}(0)=\boldsymbol{Q}_{0}, \quad \text { and } \quad \dot{\boldsymbol{Q}}(0)=\dot{\boldsymbol{Q}}_{0}
\end{gathered}
$$

where $\boldsymbol{Q}(t)$ is the generalized displacement vector, $\dot{\boldsymbol{Q}}(t)$ is the generalized velocity vector, $\ddot{\boldsymbol{Q}}(t)$ is the generalized acceleration vector, $[\mathcal{M}]$ is the mass matrix, $[\mathcal{C}]$ is the damping matrix, $[\mathcal{K}]$ is the stiffness matrix. Also, $\mathcal{F}_{N L}, \boldsymbol{Q}_{0}$, and $\dot{\boldsymbol{Q}}_{0}$ are vectors which, respectively, represent the nonlinear force, the initial generalized position, and the initial generalized velocity. This initial value problem is integrated using the Newmark method [4]. The nonlinear system of algebraic equations, resulting from the time discretization, is solved by a fixed point iteration.

\section{Numerical results}

In order to simulate the nonlinear dynamics of the mechanical system defined in the section 2.1. the physical parameters presented in the Table 3 are adopted. The dynamics is investigated during the interval of time $\left[t_{0}, t_{f}\right]=[0,200] m s$, being adopted as initial conditions a zero displacement, and a constant angular velocity $\Omega$ around the $x$ axis.

\begin{tabular}{ccl}
\hline parameter & value & unit \\
\hline$\rho$ & 7900 & $\mathrm{~kg} / \mathrm{m}^{3}$ \\
$E$ & 203 & $\mathrm{GPa}$ \\
$G$ & 78 & $\mathrm{GPa}$ \\
$R_{\text {int }}$ & 50 & $\mathrm{~mm}$ \\
$R_{\text {ext }}$ & 80 & $\mathrm{~mm}$ \\
$R_{b h}$ & 100 & $\mathrm{~mm}$ \\
$A$ & $5500 \pi$ & $\mathrm{mm}$ \\
$L$ & 100 & $\mathrm{~m}$ \\
$c$ & $1 \times 10^{-2}$ & \\
$g$ & 9.81 & $\mathrm{~m} / \mathrm{s}^{2}$ \\
$\Omega$ & 45 & $\mathrm{rpm}$ \\
$k_{F S_{1}}$ & $1 \times 10^{10}$ & $\mathrm{~N} / \mathrm{m}$ \\
$k_{F S_{2}}$ & $1 \times 10^{16}$ & $\mathrm{~N} / \mathrm{m}^{3}$ \\
$c_{F S}$ & $1 \times 10^{-3}$ & $\mathrm{~N} / \mathrm{m}^{4} \mathrm{~s}$ \\
$\mu_{F S}$ & $5 \times 10^{-4}$ & \\
\hline
\end{tabular}

Table 1: Physical parameters used in the simulation.

The discretization of the structure uses a finite element mesh with 400 elements. As each element has 6 degrees of freedom per node, this results in a semi-discrete model with 2406 degrees of freedom. For temporal integration, the numerical scheme uses a time step $\Delta t=5 \times 10^{-5} \mathrm{~s}$, which is small enough to capture the effects of shock on the dynamics.

\subsection{Transverse dynamics of the drillstring and the lateral impacts}

In this section it is analyzed the transverse dynamics of the beam, whose neutral fiber displacement in the $\mathrm{z}$ direction is presented, for some instants, in Figure 3. Illustrations of the mechanical system, sectioned by the plane $y=0$, for the same instants, can be seen in Figure 4 . 


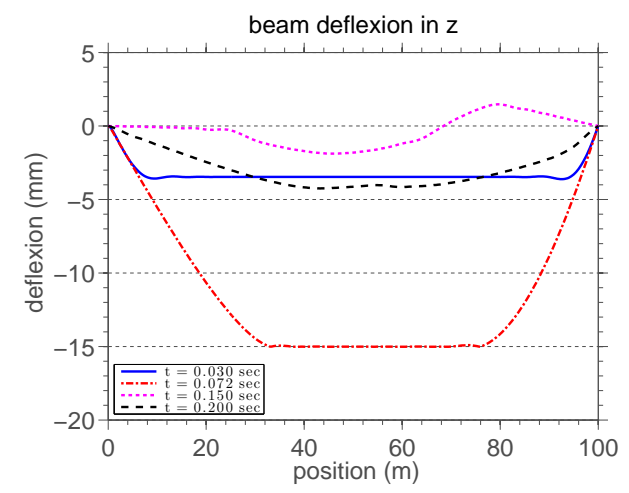

Figure 3: Beam neutral fiber displacement, in the z direction, for some instants.

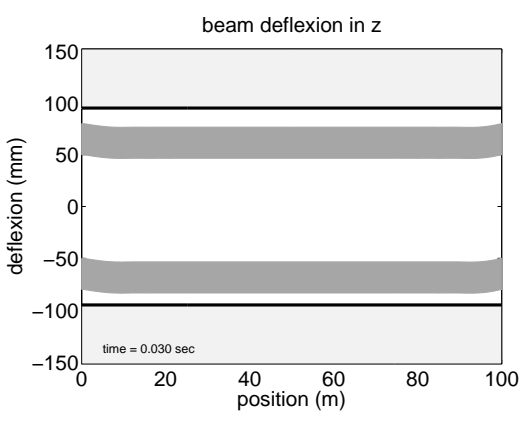

(a) $t=30 \mathrm{~ms}$

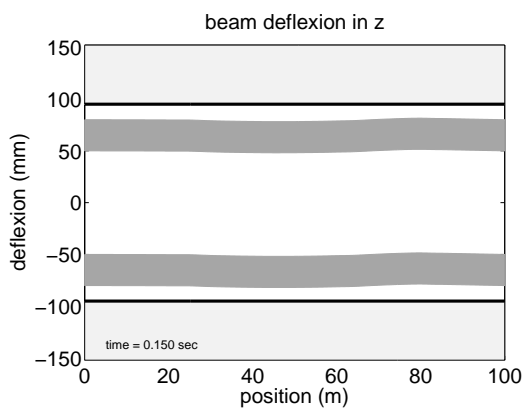

(c) $t=150 \mathrm{~ms}$

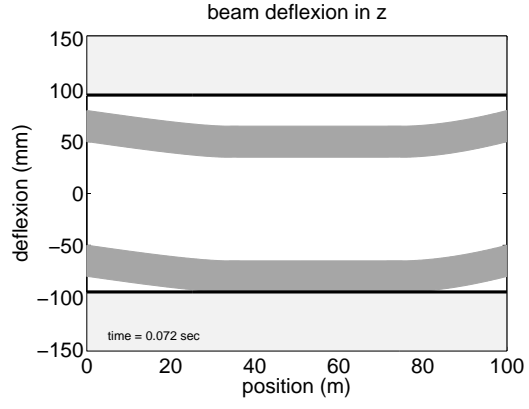

(b) $t=72 \mathrm{~ms}$

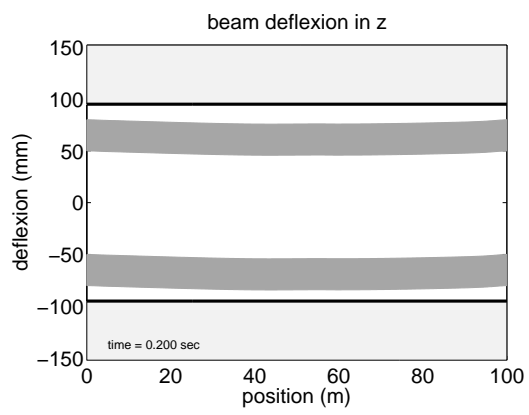

(d) $t=200 \mathrm{~ms}$

Figure 4: Illustrations of the beam, sectioned by the plane $y=0$, for the several instants.

One can note that, for none of the instants analyzed the neutral fiber assumes a parabolic shape, as occurs when a beam is subjected to its own weight. For $t=30 \mathrm{~ms}$, this is a pure consequence of the nonlinear inertial effects, induced by the rotation of the beam, combined with the geometric nonlinearity due to the large displacements. At this moment, the beam configuration is "close" to the first flexural mode, as can be seen in Figure 4(a),

However, between $t=71.8 \mathrm{~ms}$ and $t=73.6 \mathrm{~ms}$, the beam impacts the borehole wall. This situation is exemplified, for $t=72 \mathrm{~ms}$, in Figure 4(b). Note that, at this instant, the beam/wall contact is not punctual, it occurs along a line segment.

This mechanical interaction between the two bodies generates a nonlinear elastic deformation in both bodies, but without residual deformation effects. In this contact also occurs energy dissipation, due to the normal shock, and the torsional friction, induced by the rotation of the beam. These nonlinear effects have consequences on subsequent dynamic behavior of the mechanical system. 
In the instants that follow, additional impacts do not occur, and the beam assumes configurations that are "close" to flexural modes of order higher than one, as can be see in Figures $4(\mathrm{c})$ and $4(\mathrm{~d})$.

\section{Final remarks}

In this work it was discussed the the effects induced by lateral shock in nonlinear dynamic behavior of a annular beam that emulates a drillstring in horizontal configuration. The numerical simulations results show that the shock significantly modifies the dynamics of the mechanical system, leading to the appearance of configurations "close" to high-order flexural modes.

The mathematical model developed in this work is subject to uncertainties, that are due to the variability of its parameters, and/or epistemic in nature, i.e, result of the ignorance about the physics of the problem [9]. For this reason, in a future work, the authors intend to quantify the uncertainties of the model using a nonparametric probabilistic approach [10] to construct a stochastic model.

\section{Acknowledgments}

The authors are indebted to the Brazilian agencies CNPq, CAPES, and FAPERJ for the financial support given to this research.

\section{References}

[1] A. Cunha Jr. Modeling and Uncertainty Quantification in the Nonlinear Stochastic Dynamics of Horizontal Drillstrings. D.Sc. Thesis, Pontifícia Universidade Católica do Rio de Janeiro, Rio de Janeiro.

[2] A. Cunha Jr, C. Soize, and R. Sampaio. Computational modeling of the nonlinear stochastic dynamics of horizontal drillstrings. (in preparation).

[3] G. Gilardi and I. Sharf. Literature survey of contact dynamics modelling. Mechanism and Machine Theory, 37:1213-1239, 2002. doi:10.1016/S0094-114X(02)00045-9.

[4] T. J. R. Hughes. The Finite Element Method. Dover Publications, New York, 2000.

[5] K. H. Hunt and F. E. Crossley. Coefficient of restitution interpreted as damping in vibroimpact. Journal of Applied Mechanics, 42:440-445, 1975. doi:10.1115/1.3423596.

[6] T. G. Ritto, M. R. Escalante, R. Sampaio, and M. B. Rosales. Drill-string horizontal dynamics with uncertainty on the frictional force. Journal of Sound and Vibration, 332:145153, 2013. doi:10.1016/j.jsv.2012.08.007.

[7] T. G. Ritto, C. Soize, and R. Sampaio. Non-linear dynamics of a drill-string with uncertain model of the bit-rock interaction. International Journal of Non-Linear Mechanics, 44:865876, 2009. doi:10.1016/j.ijnonlinmec.2009.06.003.

[8] R. Sampaio, M.T. Piovan, and G. Venero Lozano. Coupled axial/torsional vibrations of drill-strings by means of non-linear model. Mechanics Research Communications, 34:497502, 2007. doi:10.1016/j.mechrescom.2007.03.005.

[9] G. I. Schuëller. On the treatment of uncertainties in structural mechanics and analysis. Computers \&5 Structures, 85:235-243, 2007. 
[10] C. Soize. Stochastic modeling of uncertainties in computational structural dynamics Recent theoretical advances. Journal of Sound and Vibration, 332:2379-2395, 2013. doi: $10.1016 /$ j.jsv.2011.10.010.

[11] P. D. Spanos, A. M. Chevallier, and N. P. Politis. Nonlinear stochastic drill-string vibrations. Journal of Vibration and Acoustics, 124:512-518, 2002. doi:10.1115/1.1502669.

[12] P. D. Spanos, A. M. Chevallier, N. P. Politis, and M. L. Payne. Oil and gas well drilling: a vibrations perspective. The Shock and Vibration Digest, 35:85-103, 2003.

[13] M. A. Trindade, C. Wolter, and R. Sampaio. Karhunen-Loève decomposition of coupled axial/bending vibrations of beams subject to impacts. Journal of Sound and Vibration, 279:1015-1036, 2005. doi:10.1016/j.jsv.2003.11.057. 